\title{
The long and short of cephalomedullary nails in the treatment of osteoporotic pertrochanteric fracture
}

\author{
Choon Chiet Hong $^{1}$, MBBS, MRCSE, Nazrul Nashi ${ }^{1}$, MBBS, MRCSE, Milindu Chanaka Makandura ${ }^{1}$, MBBS, \\ Jiong Hao Jonathan $\underline{\text { Tan }}^{1}$, MBBS, MRCS, Luke Peter $^{1}$, MBBS, BMedSci, Diarmuid Murphy ${ }^{1}$, MB BCh BAO, FRCS
}

INTRODUCTION Pertrochanteric fractures after low-energy trauma are common among osteoporotic patients. Although the use of intramedullary devices to treat such fractures is becoming increasingly popular, there is a paucity of data comparing the outcomes of the use of short cephalomedullary nails (SCN) with the use of long cephalomedullary nails (LCN). This study aimed to compare the outcomes of treatment using LCN with treatment using SCN for patients with osteoporotic pertrochanteric fractures.

METHODS A retrospective review of 64 patients with osteoporotic pertrochanteric fractures who were treated with either LCN or SCN and had a minimum follow-up of one year was performed. Primary outcome measures include complications, revision surgeries and union rates. Secondary outcome measures include duration of surgery, estimated blood loss, length of hospital stay, and ambulatory and mortality status at one year.

RESULTS There was no significant difference in the clinical and functional outcomes of the patients who were treated with LCN and those who were treated with SCN. However, there was a higher incidence of heterotopic ossification in the latter group, and a slightly greater average estimated blood loss and duration of surgery in the former group. Patients treated with LCN tended to be more osteoporotic.

CONCLUSION Our study found no significant difference in terms of complications, revision surgeries, union rates and ambulatory status between the patients who were treated with LCN and those who were treated with SCN. Both LCN and SCN provided safe and reliable outcomes in the treatment of osteoporotic pertrochanteric fractures.

Keywords: cephalomedullary nails, complications, osteoporosis, pertrochanteric fracture, short versus long nail

\section{INTRODUCTION}

Osteoporosis is a significant condition affecting the global population, particularly the female gender. ${ }^{(1)}$ It is associated with many complications, such as hip fracture. Studies have projected that by 2050 , potentially $50 \%$ of all hip fractures in the world will occur in Asia and Latin America, where osteoporosis is most prevalent. ${ }^{(2)}$ As hip fractures are associated with substantial global morbidity and loss of disability-adjusted life years, ${ }^{(3)}$ it is imperative to take steps to counter the impending epidemic by improving preventive and pharmacological measures against osteoporosis and fragility fractures. This allows patients the possibility of achieving their premorbid status and restoring their quality of life.

Patients with osteoporosis commonly develop pertrochanteric fractures after low-energy trauma. Surgical fixation of such fractures in osteoporotic bone is difficult, but both extra- and intramedullary devices have been utilised with good outcomes. ${ }^{(4,5)}$ There is, however, a lack of studies demonstrating the superiority of either type of device. Several authors have reported an increasing trend in the use of intramedullary devices to fix pertrochanteric fractures in patients with osteoporosis, despite the lack of supporting evidence. ${ }^{(4,6-9)}$ Nonetheless, intramedullary fixation with both long and short cephalomedullary nails has been utilised with good clinical outcomes. ${ }^{(4,5,10-13)}$

There are concerns regarding the use of long and short cephalomedullary nails in intramedullary fixation. Some authors believe that short nails provide inadequate diaphyseal fixation as compared to long nails, especially with subtrochanteric fracture extensions. ${ }^{(14)}$ Furthermore, there is a risk of developing stress fractures at the tips of short nails, particularly in osteoporotic bone. ${ }^{(15,16)}$ On the other hand, the use of long nails is more expensive and requires a longer operation time due to the need for intramedullary canal reaming. ${ }^{(4,14,15)}$

Although some authors have suggested that cephalomedullary nailing is superior for pertrochanteric fractures with a subtrochanteric extension (i.e. Orthopaedic Trauma Association [OTA] Class 31-A3 fractures), ${ }^{(17,18)}$ simple and multifragmentary pertrochanteric fractures without a subtrochanteric extension (i.e. OTA Class 31-A1 and Class 31-A2 fractures) continue to be treated with both long and short cephalomedullary devices. Based on our literature review, only three studies have attempted to compare the outcomes of the use of long cephalomedullary nails with the use of short cephalomedullary nails in the management of OTA Class 31-A1 and Class 31-A2 pertrochanteric fractures. ${ }^{(14,19,20)}$ These studies were largely of elderly populations, which have a high probability of underlying osteoporosis based on their age alone. However, none of these studies employed objective evaluation tools to diagnose and study the extent of osteoporosis. Therefore, the present study aimed to compare the complication rates and functional outcomes of treatment using long cephalomedullary nails with treatment using short cephalomedullary nails, for osteoporotic patients with OTA Class 31-A1 and Class 31-A2 pertrochanteric fractures. 


\section{METHODS}

A retrospective review of all patients admitted for operative fixation of pertrochanteric fractures with cephalomedullary nailing at National University Hospital, Singapore, from July 2009 to July 2012 was conducted. The patients were identified using the hospital's diagnosis and operative code system. The patients' case notes, electronic records and radiographs were reviewed. This study was approved by the local Institutional Review Board.

All patients aged $\geq 21$ years with simple and multifragmentary pertrochanteric fractures without a subtrochanteric extension (i.e. OTA Class 31-A1 and Class 31-A2 fractures), and a bone mineral density (BMD) T-score $<-2.5$ (i.e. the patient was osteoporotic) were eligible for inclusion in the study. Included patients had also completed at least one year of follow-up. Patients who were skeletally immature and those who had unstable fractures (i.e. OTA Class 31-A3), subtrochanteric extensions, open fractures, concomitant fractures and/or neurovascular injury in the ipsilateral lower limb, pathological fractures, and previous hip infections or surgery were excluded. Pertinent patient demographics (e.g. age, gender, comorbidities and American Society of Anesthesiologists [ASA] score), mechanism of injury, fracture type, BMD T-score, operative details (e.g. implant used, duration of surgery and estimated blood loss), pre- and post-injury ambulatory status, length of hospital stay (LOS), postoperative radiographs and mortality rate were extracted and analysed statistically.

The fractures were categorised radiographically according to the OTA classification. ${ }^{(21)}$ The ASA score was used to assess the severity of the patients' health problems at the time of presentation. ${ }^{(22)}$ The mechanism of injury was classified into high-velocity injuries, which included road traffic accidents and falls from a height greater than the patient's own standing height, and low-velocity injuries, which included direct contusions and falls from a height equal or less than the patient's own standing height. The assessment of BMD was based on the dual energy X-ray absorptiometry (DEXA) tool. Based on the World Health Organization's definition of osteoporosis, a BMD T-score of: $>-1$ is normal, $\leq-1$ to $\geq-2.5$ indicates osteopenia and $<-2.5$ signifies osteoporosis. ${ }^{(16)}$ Estimated blood loss was defined as the maximum drop in postoperative haemoglobin $(\mathrm{Hb})$ level, as measured on postoperative Day 1. Pre- and post-injury ambulatory status included the following categories: independent ambulators; ambulators with walking aids; and non-ambulators. LOS was measured in days, from time of admission to time of discharge.

Primary outcome measures of this study included surgical complications (e.g. periprosthetic fracture, nonunion, implant cutout, screw/blade backout, infection, development of heterotopic ossification, medialisation of the femoral shaft and intraoperative fracture of the lateral wall), the need for revision surgeries and union rates. Secondary outcome measures included duration of surgery, estimated blood loss, LOS, and ambulatory and mortality status at one year. Surgical complications were diagnosed on serial radiographic monitoring. Heterotopic ossification was defined as abnormal formation of mature, lamellar bone in nonosseous tissues. Bony union was defined as complete cortical bridging between proximal and distal fragments with no visible fracture line at one year.

All surgeries were performed by fellowship-trained orthopaedic consultants and specialist registrars. The surgeries were performed with fluoroscopic guidance and with the patient under anaesthesia. A single implant design was utilised in our hospital: the proximal femoral nail antirotation (PFNA) nail (Synthes GmbH, Oberdorf, Switzerland). After closed reduction using fluoroscopic guidance, the same operative technique was used for all cases, utilising the cannulated opening reamer, guidewire placement, reaming to the appropriate size and insertion of appropriately sized nails, followed by placement of the helical blade. An accompanying jig was used to guide the insertion of the helical blade and distal interlocking screw. Although distal interlocking screws were inserted for all short nails, the decision to insert distal interlocking screws for the long nails differed according to the surgeon's preference; the decision was made intraoperatively, based on the characteristics of the fracture and patient factors. After the operation, all patients were allowed to bear weight as tolerated. They were reviewed two weeks after the operation for wound inspection and suture removal if needed. Subsequent reviews were at six weeks, and three, six and 12 months after the operation. Standard radiography of the hip was performed at these reviews.

Frequency tables and descriptive statistics (i.e. mean, standard deviation or median, when appropriate) were presented for all variables. Categorical variables were presented as proportions, while continuous variables were presented as means or medians. Chi-squared test was used for comparison between categorical variables, while Student's $t$-test and Mann-Whitney $U$ test were used for continuous variables. Statistical significance was accepted if the $p$-value was $<0.05$. Data analysis was performed using SPSS version 16.0 (SPSS Inc, Chicago, IL, USA).

\section{RESULTS}

A total of 64 patients (45 female, 19 male) fulfilled the inclusion criteria of this study. Their mean age was 79.9 (range 56-97) years and 38 of them had a left-sided hip injury. Based on the OTA classification, 15 patients had OTA Class 31-A1 fractures, while 49 had OTA Class 31-A2 fractures. All of the patients were osteoporotic, with an average T-score of -3.45 (range -2.6 to -5.4$)$. Most of the patients $(n=62)$ had low-velocity injuries. In terms of ASA grading, two patients were categorised as ASA Grade 1, 31 as ASA Grade 2 and 31 as ASA Grade 3. 20 patients were treated with long PFNA nails and 44 were treated with short PFNA nails. The long nails were $320 \mathrm{~mm}(\mathrm{n}=1), 340 \mathrm{~mm}$ $(n=11)$ or $380 \mathrm{~mm}(n=8)$ in length, while all short nails were $200 \mathrm{~mm}$ in length. The diameter of the nails ranged from $9 \mathrm{~mm}$ to $12 \mathrm{~mm}$ : most were $10 \mathrm{~mm}(\mathrm{n}=28)$, followed by $11 \mathrm{~mm}(\mathrm{n}=21)$, $12 \mathrm{~mm}(\mathrm{n}=10)$ and $9 \mathrm{~mm}(\mathrm{n}=5)$. Distal interlocking screws were used with both short and long PFNA nails. In terms of preinjury ambulatory status, 41 of the patients were independent ambulators, 20 were ambulators with walking aids and three were non-ambulators. Patient demographics and clinical data are shown in Table I. No significant differences, in terms of 
demographics and pre-injury mobility status, were found between the patients with short PFNA nails and those with long PFNA nails.

In this study, the overall complication rate was $26.6 \%$, i.e. complications occurred in 17 of the 64 patients. The overall complication rate represented the estimated risk of developing at least one local postoperative complication and was based on the overall number of patients and not the total number of complications. If a patient experienced multiple complications under any complication class, the patient was only counted once. The number of patients with each class of complication is shown in Table II. Five cases required revision surgery - one involved a patient who was treated with a long PFNA nail, while four involved patients who were treated with short PFNA nails. In the case involving a long nail, nonunion and blade backout was observed, and the patient's surgery was revised to a bipolar hemiarthroplasty. In the four cases involving short nails, there were two cases of periprosthetic fractures (one case was revised to long nail and the other to cerclage wiring), one case of distal femur supracondylar fracture (distal plate and screw construct was done) and one case of nonunion with blade backout (the blade was consequently removed) (Fig. 1). Although the distal femur supracondylar fracture occurred several months after the operation via an accidental mechanical fall and was nowhere near the tip of the short nail, it was taken as a complication as well. When we compared the use of long PFNA nails with that of short PFNA nails in the treatment of osteoporotic pertrochanteric fractures, we found no significant differences in terms of complication rate and the need for revision surgery $(p=0.689)$. This was despite the higher incidence of heterotopic ossification observed among patients who were treated with short PFNA nails (Table II).

The average preoperative and postoperative $\mathrm{Hb}$ level was $11.1 \mathrm{~g} / \mathrm{dL}$ and $8.8 \mathrm{~g} / \mathrm{dL}$, respectively. An average drop of 2.2 (range $0.1-6.0) \mathrm{g} / \mathrm{dL}$ in Hb level was observed in our study cohort. No significant difference was observed in the estimated blood loss (i.e. maximum drop in $\mathrm{Hb}$ postoperatively) between the patients treated with long PFNA nails and those treated with short PFNA nails ( $2.6 \mathrm{~g} / \mathrm{dL}$ vs. $2.1 \mathrm{~g} / \mathrm{dL}$, respectively; $\mathrm{p}=0.150)$, although it was slightly higher among the patients treated with long PFNA nails. The average duration of surgery was 76.6 (range 29-315) minutes. No significant difference was noted in the average duration of surgery for the patients treated with long or short PFNA nails (78.2 minutes vs. 73.0 minutes, respectively; $p=0.617$ ). In addition, there was no significant difference between the median LOS of patients treated with long PFNA nails and that of patients treated with short PFNA nails (14.0 days vs. 15.5 days, respectively; $p=0.793$ ). On the other hand, patients treated with long PFNA nails tended to be more osteoporotic than those treated with short PFNA nails (T-score: -3.79 vs $-3.29 ; p=0.08$ ) (Table III).

The mobility of the patients after fracture fixation was also reviewed and analysed (Table IV). Among the 41 patients who were able to ambulate independently prior to their injury, 23 were dependent on walking aids one year after the operation; among the 20 patients who were ambulatory with walking aids prior to their injury, eight were non-ambulant one year after the operation $(p=0.011)$. There was no significant difference
Table I. Demographic and clinical data of the patients $(n=64)$.

\begin{tabular}{|c|c|c|c|}
\hline \multirow[t]{2}{*}{ Variable } & \multicolumn{2}{|c|}{ No. (\%) } & \multirow[t]{2}{*}{ p-value } \\
\hline & $\begin{array}{l}\text { Short nail } \\
(n=44)\end{array}$ & $\begin{array}{l}\text { Long nail } \\
(n=20)\end{array}$ & \\
\hline Age* (yr) $^{*}$ & $80.0(60-93)$ & $79.8(56-97)$ & 0.920 \\
\hline Gender & & & 0.971 \\
\hline Female & $31(70.5)$ & $14(70.0)$ & \\
\hline Male & $13(29.5)$ & $6(30.0)$ & \\
\hline Side injured & & & 0.537 \\
\hline Left & $25(56.8)$ & $13(65.0)$ & \\
\hline Right & $19(43.2)$ & $7(35.0)$ & \\
\hline OTA classification & & & 0.662 \\
\hline $31-A 1$ & $11(25.0)$ & $4(20.0)$ & \\
\hline $31-A 2$ & $33(75.0)$ & $16(80.0)$ & \\
\hline ASA grade & & & 0.813 \\
\hline 1 & $1(2.3)$ & $1(5.0)$ & \\
\hline 2 & $22(50.0)$ & $9(45.0)$ & \\
\hline 3 & $21(47.7)$ & $10(50.0)$ & \\
\hline Mechanism of injury & & & 0.625 \\
\hline Low velocity & $42(95.5)$ & $20(100.0)$ & \\
\hline High velocity & $2(4.5)$ & $0(0)$ & \\
\hline $\begin{array}{l}\text { Pre-injury mobility } \\
\text { status }\end{array}$ & & & 0.579 \\
\hline $\begin{array}{l}\text { Independent } \\
\text { ambulator }\end{array}$ & $30(68.2)$ & $11(55.0)$ & \\
\hline $\begin{array}{l}\text { Ambulator with } \\
\text { walking aid }\end{array}$ & $12(27.3)$ & $8(40.0)$ & \\
\hline Non-ambulator & $2(4.5)$ & $1(5.0)$ & \\
\hline
\end{tabular}

*Data presented as mean (range). ASA: American Society of Anesthesiologists; OTA: Orthopaedic Trauma Association

Table II. Types of complications observed in patients with short or long proximal femoral nail antirotation devices.

\begin{tabular}{lcc}
\hline \multirow{2}{*}{ Complication } & \multicolumn{2}{c}{ No. (\%) } \\
\cline { 2 - 3 } & Short nail ( $\mathbf{n}=\mathbf{4 4 )}$ & Long nail $(\mathbf{n}=\mathbf{2 0})$ \\
\hline Heterotopic ossification & $5(11.4)$ & $1(5.0)$ \\
Medialisation & $5(11.4)$ & $2(10.0)$ \\
Lateral wall fracture & $1(2.3)$ & $0(0)$ \\
Wound infection & $1(2.3)$ & $0(0)$ \\
Nonunion & $1(2.3)$ & $1(5.0)$ \\
Blade backout & $1(2.3)$ & $1(5.0)$ \\
Periprosthetic fracture & $3(6.8)$ & $0(0)$ \\
\hline
\end{tabular}

in ambulatory status at one year between the patients treated with long PFNA nails and those treated with short PFNA nails $(p=0.155)$ (Table III). Among the patients treated with long PFNA nails, six of the 11 patients who were independent ambulators prior to their injury remained independent ambulators one year after the operation $(p=0.043)$. In contrast, among the patients treated with short PFNA nails, only four of the 30 patients who were independent ambulators prior to their injury remained independent ambulators one year after the operation; however, this was not statistically significant $(p=0.145)$. In terms of mortality, two of the 64 patients died - one was an inpatient death due to ischaemic bowel, while the other was due to pneumonia after one 

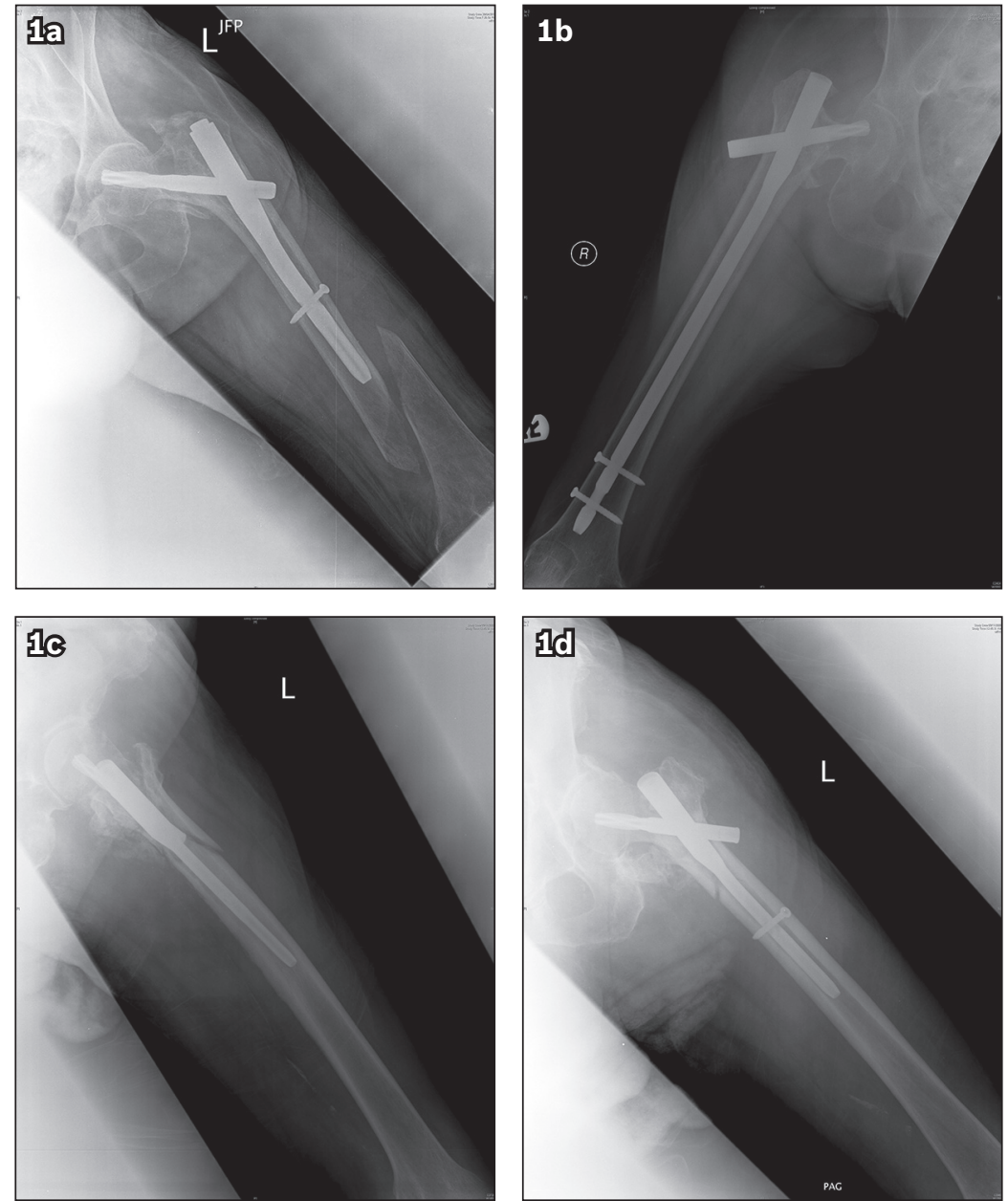

Fig. 1 Radiographs show (a) a periprosthetic fracture at the tip of a short proximal femoral nail antirotation (PFNA) nail; (b) nonunion of the fracture and PFNA blade backout; and (c \& d) a periprosthetic fracture just below the trochanter with the nail still well fixed.

Table III. Comparison between patients treated with short and long proximal femoral nail antirotation devices.

\begin{tabular}{|c|c|c|c|}
\hline \multirow[t]{2}{*}{ Variable } & \multicolumn{2}{|c|}{ Mean (range) } & \multirow[t]{2}{*}{ p-value } \\
\hline & Short nail $(n=44)$ & Long nail $(n=20)$ & \\
\hline Preoperative $\mathrm{Hb}(\mathrm{g} / \mathrm{dL})$ & $10.9(7.4-14.4)$ & $11.4(8.8-15.2)$ & 0.198 \\
\hline Postoperative $\mathrm{Hb}$ (g/dL) & $8.8(6.1-11.4)$ & $8.8(6.7-11.4)$ & 0.999 \\
\hline Estimated blood loss (g/dL) & $2.1(0.1-5.6)$ & $2.6(0.1-6.0)$ & 0.150 \\
\hline Duration of surgery (min) & $73.0(40-121)$ & $78.2(29-315)$ & 0.617 \\
\hline $\operatorname{LOS}^{*}$ (day) & $15.5(4-53)$ & $14.0(3-30)$ & 0.793 \\
\hline T-score & $-3.29(-5.4$ to -2.6$)$ & $-3.79(-5.4$ to -2.6$)$ & 0.08 \\
\hline Ambulatory status at $1 \mathrm{yr}^{\dagger}$ & & & 0.155 \\
\hline Independent ambulator & $4(9.1)$ & $6(30.0)$ & \\
\hline Ambulator with walking aid & $26(59.1)$ & $8(40.0)$ & \\
\hline Non-ambulator & $13(29.5)$ & $6(30.0)$ & \\
\hline Deceased & $1(2.3)$ & $0(0)$ & \\
\hline Mortality & $2(4.5)$ & $0(0)$ & 0.625 \\
\hline
\end{tabular}

*Data presented as median (range). ${ }^{\dagger}$ Data presented as no. (\%). Hb: haemoglobin; LOS: length of hospital stay

year. There was no significant difference in the mortality rates of the patients treated with long PFNA nails and those treated with short PFNA nails (Table III).

When the patients were stratified according to their fracture type (i.e. OTA Class 31-A1 vs. OTA Class 31-A2), we found no significant differences between the patients with OTA Class 31-A1 fractures and those with OTA Class 31-A2 fractures, in terms of their age, gender, ASA grade, mechanism of injury, estimated blood loss, duration of surgery, LOS, degree of osteoporosis, choice of short or long PFNA nail, number of postoperative complications, and mobility and mortality rate. However, the patients with OTA Class 31-A1 fractures were younger at presentation ( 77.5 years vs. 80.7 years) and had a lower volume of estimated blood loss (1.9 g/dL vs. $2.3 \mathrm{~g} / \mathrm{dL})$ than the patients 
Table IV. Pre-injury ambulatory status and change in ambulation after pertrochanteric fracture fixation.

\begin{tabular}{|c|c|c|c|c|}
\hline \multirow[t]{2}{*}{ Ambulatory status at $1 \mathrm{yr}$} & \multicolumn{3}{|c|}{ Pre-injury ambulatory status (No. [\%]) } & \multirow[t]{2}{*}{ p-value } \\
\hline & Independent ambulator $(n=41)$ & Ambulator with walking aid $(n=20)$ & Non-ambulator $(n=3)$ & \\
\hline Independent ambulator & $10(24.4)$ & $0(0)$ & $0(0)$ & 0.011 \\
\hline Ambulator with walking aid & $23(56.1)$ & $11(55.0)$ & $0(0)$ & \\
\hline Non-ambulator & $8(19.5)$ & $8(40.0)$ & $3(100.0)$ & \\
\hline Deceased & $0(0)$ & $1(5.0)$ & $0(0)$ & \\
\hline
\end{tabular}

Table V. Comparison between the fracture types $(n=64)$.

\begin{tabular}{|c|c|c|c|}
\hline \multirow[t]{2}{*}{ Variable } & \multicolumn{2}{|c|}{ No. (\%) } & \multirow[t]{2}{*}{ p-value } \\
\hline & OTA Class 31-A1 ( $\mathrm{n}=15)$ & OTA Class 31-A2 $(n=49)$ & \\
\hline Age* (yr) $^{*}$ & $77.5(60-88)$ & $80.7(56-97)$ & 0.196 \\
\hline Gender & & & 0.113 \\
\hline Female & $2(13.3)$ & $17(34.7)$ & \\
\hline Male & $13(86.7)$ & $32(65.3)$ & \\
\hline ASA grade & & & 0.666 \\
\hline 1 & $1(6.7)$ & $1(2.0)$ & \\
\hline 2 & $7(46.7)$ & $24(49.0)$ & \\
\hline 3 & $7(46.7)$ & $24(49.0)$ & \\
\hline Mechanism of injury & & & 0.165 \\
\hline Low velocity & $14(93.3)$ & $48(98.0)$ & \\
\hline High velocity & $1(6.7)$ & $1(2.0)$ & \\
\hline Estimated blood loss* (g/dL) & $1.9(0.1-5.5)$ & $2.3(0.1-6.0)$ & 0.309 \\
\hline Duration of surgery $(\mathrm{min})$ & $77.6(29-127)$ & $76.2(36-315)$ & 0.901 \\
\hline $\operatorname{LOS}^{\dagger}($ day) & $13(4-53)$ & $16(3-33)$ & 0.608 \\
\hline T-score ${ }^{*}$ & $-3.48(-5.4$ to -2.8$)$ & $-3.44(-5.4$ to -2.6$)$ & 0.854 \\
\hline Length of nail & & & 0.662 \\
\hline Short & $4(26.7)$ & $16(32.7)$ & \\
\hline Long & $11(73.3)$ & $33(67.3)$ & \\
\hline Complication & & & 0.459 \\
\hline Heterotopic ossification & $0(0)$ & $6(12.2)$ & \\
\hline Medialisation & $1(6.7)$ & $6(12.2)$ & \\
\hline Lateral wall fracture & $0(0)$ & $1(2.0)$ & \\
\hline Wound infection & $0(0)$ & $1(2.0)$ & \\
\hline Nonunion & $1(6.7)$ & $1(2.0)$ & \\
\hline Blade backout & $1(6.7)$ & $1(2.0)$ & \\
\hline Periprosthetic fracture & $1(6.7)$ & $2(4.1)$ & \\
\hline Ambulatory status at $1 \mathrm{yr}$ & & & 0.644 \\
\hline Independent ambulator & $2(13.3)$ & $8(16.3)$ & \\
\hline Ambulator with walking aid & $10(66.7)$ & $24(49.0)$ & \\
\hline Non-ambulator & $3(20.0)$ & $16(32.7)$ & \\
\hline Deceased & $0(0)$ & $1(2.0)$ & \\
\hline Mortality & $0(0)$ & $2(4.1)$ & 0.427 \\
\hline
\end{tabular}

*Data presented as mean (range). ${ }^{\dagger}$ Data presented a s median (range). ASA: American Society of Anesthesiologists; LOS: length of hospital stay, OTA: Orthopaedic Trauma Association

with OTA Class 31-A2 fractures, while the patients with OTA Class 31-A2 fractures were found to be more likely to develop heterotopic ossification and medialisation; these observations were, however, not statistically significant (Table V).

\section{DISCUSSION}

Managing patients with fragility fractures such as pertrochanteric fractures has always been a challenge for orthopaedic surgeons.
These fractures are commonly managed with cephalomedullary nails despite the lack of evidence, especially regarding the choice of long or short nails..$^{(4,5,10-13,20)}$ Several studies have reported no significant difference in outcome with the use of long or short intramedullary nails to treat simple and multifragmentary pertrochanteric fractures; however, those studies did not consider the presence of osteoporosis in their analysis. ${ }^{(14,19)}$ Hence, the aim of the present study was to review the complications and functional 
outcomes of the use of long versus short cephalomedullary nails for the management of osteoporotic patients with pertrochanteric fractures, specifically OTA Class 31-A1 and Class 31-A2 fractures.

In recent years, there has been growing concern regarding the high risk of implant cut-out in the management of osteoporotic pertrochanteric fractures, as was aptly described by Konstantinidis et al in their cadaveric study. ${ }^{(23)}$ In the present study, there were no significant differences in complication, revision and union rates between the patients who were treated with long cephalomedullary nails and those treated with short cephalomedullary nails for osteoporotic pertrochanteric fractures. This finding is in keeping with other studies in the literature. For example, a study by Hou et al, which reviewed 283 patients who underwent either short or long cephalomedullary nail insertion for pertrochanteric fractures (i.e. OTA Class 31-A1 and Class 31-A2 fractures), reported no significant difference in the complication or union rates between the two groups. ${ }^{(14)}$ Although Hou et al attempted to compare osteoporotic and non-osteoporotic patients in their study, they did not clarify how osteoporosis was diagnosed or provide further information about its severity. ${ }^{(14)}$ Other than the study by Hou et al, Boone et al also showed that there was no significant difference in the LOS and incidence of periprosthetic fracture of patients treated with long and short nails. ${ }^{(19)}$ Boone et al's study, which was a review of 194 patients with intertrochanteric fractures (i.e. OTA Class 31-A1 and Class 31-A2 fractures) treated with long or short nails, did not review whether the patients in their study cohort were osteoporotic. ${ }^{(19)}$ Following that, Kleweno et al's review of 559 patients also did not find any significant difference between the failure rates of short and long cephalomedullary nails used for the treatment of intertrochanteric hip fractures. ${ }^{(20)}$ The study similarly did not discuss whether the patients had osteoporosis and the severity of the disease, or compare how osteoporotic patients differed from non-osteoporotic patients in terms of the outcome of the use of short or long cephalomedullary nails. However, the study consisted of patients over the age of 65 years. ${ }^{(20)}$ It was hence likely that a large proportion of the patients were osteoporotic, although no specific diagnostic tools were used to diagnose osteoporosis.

Despite the additional risk of periprosthetic fracture at the tip of short nails, particularly in osteoporotic bones, the study found no difference in the development of periprosthetic fractures between long and short cephalomedullary nails, although all three cases of periprosthetic fracture occurred in the short nail group. Of the three periprosthetic fractures, the fracture was distal to the tip of the short nail in only one case, while the other two cases were fractured around the trochanteric region and the distal femur supracondylar region. Previous studies have hypothesised that short nails were more likely to create diaphyseal stress risers at the tip, causing periprosthetic fractures. ${ }^{(15,16)}$ However, newer implants with better designs, such as lower modulus elasticity (e.g. titanium), more anatomical femoral bow, tapered tips and smaller distal locking screws, have been developed to address this issue. It is therefore interesting to note the marked difference in result between Konstantinidis et al's in vitro studies compared to clinical outcomes; it has been postulated that the interplay of the body's supporting structures (e.g. muscles and tendons) and newer implant designs play a pivotal role in reducing such complications. ${ }^{(23)}$

The present study did not find any significant difference in the duration of surgery and estimated blood loss between patients who were treated with long cephalomedullary nails and patients who were treated with short cephalomedullary nails, even though the average duration of surgery and estimated blood loss were slightly higher among the former group. This finding is in contrast to the findings of Hou et al and Boone et al. ${ }^{(14,19)}$ Several authors attributed the increase in surgical time and estimated blood loss among patients who had insertions of long cephalomedullary nails to additional reaming of the femoral canal and the insertion of the distal interlocking screw. . $^{(4,14,20)}$ We are in agreement with these postulations, especially in a setting where the use of short nails allows the additional benefit of an accompanying jig on which to place the distal interlocking screw, making the procedure easier and faster. The differences between our study and other studies that we observed could be due to our relatively smaller study population and inherent heterogeneity in the surgeons' individual implantation techniques. However, we feel that this study reflects the true circumstances of surgical care for our cohort of osteoporotic patients, rather than the experiences of a single surgeon or a selected few.

It is well known that a large proportion of patients who undergo hip fracture surgeries have poor functional status, reduced ambulation, impaired activities of daily living and significant mortality after the surgery. ${ }^{(2,3,24-26)}$ In a study by Formiga et al, only $16 \%$ of the patients could walk independently after hip fracture surgery, although 54\% were able to walk independently prior to their hip fracture, and up to $45 \%$ of the patients were housebound after hip surgery, although only $11 \%$ were housebound prior to their injury. ${ }^{(24)}$ In the present study, we found that a notable proportion of the patients were able to stay ambulant one year after the surgery, although not at their pre-injury level. After the surgery, more than half of the patients who could ambulate independently prior to their hip injury had to use walking aids, and almost half of those who required walking aids prior to their hip injury were non-ambulant (i.e. wheelchair or bedbound). In terms of treatment modality, we found no significant difference in ambulatory status at one year after surgery between the osteoporotic patients who were treated with short nails and those who were treated with long nails, even though more patients in the former group were ambulatory with walking aids at one year after surgery as compared to the latter group. We did not observe any significant difference in the mortality rate at one year between the osteoporotic patients who were treated with short nails and those who were treated with long nails.

As pointed out in the paper by Konstantinidis et al, bone quality, surgical technique and implant design are key factors in determining outcomes of pertrochanteric fracture stabilisation. ${ }^{(23)}$ In their cadaveric study, Konstantinidis et al found 
that the risk of implant cut-out/fixation failure was 11 times greater in osteoporotic bone. ${ }^{(23)}$ In the present study and those found in the literature, the presence of osteoporosis did not determine the type of nails used. In fact, to date, there has been no evidence to demonstrate which type of nail is more suitable for osteoporotic patients. In this study, however, we found that the T-scores of the patients who were treated with long nails tend to be lower than those of the patients treated with short nails (-3.79 vs. -3.29 ; $p=0.08$ ). We postulate that this is possibly due to the thinner cortices, widened medullary canal and osteopenia seen on plain radiographs prior to fixation, which influence surgeons' decision to select longer nails to achieve stability. This may also be due to the inherent belief that short nails may create a diaphyseal stress riser, resulting in distal nail tip periprosthetic fracture. ${ }^{(15,16)}$ This leads to plausible selection bias when choosing the nail length for osteoporotic pertrochanteric fractures. Managing osteoporotic bone itself is a challenge for surgeons due to the inherently low BMD and distorted microarchitecture, as well as the possible stressors placed on it by the implant. Larger prospective studies should be conducted to evaluate the use of cephalomedullary implants on osteoporotic bones before a particular implant is deemed more suitable than another, and to determine whether a more 'osteoporotic bone-friendly' design should be considered for use in osteoporotic patients.

The limitations of this study include its retrospective nature, the relatively smaller cohort of patients, lack of a longer-term follow-up and that the results generated were from a single centre. Additionally, as the choice of using a short nail or a long nail was based on the surgeons' preference, selection bias may have been present. The retrospective nature of the study meant that it was limited by the inherent heterogeneity of the surgeons' individual implantation techniques. This, however, reflects the reality of surgical care in many institutions. Despite the aforementioned limitations, the study analysed only osteoporotic fractures that were diagnosed using the DEXA tool and had a T-score $<-2.5$. Furthermore, only OTA Class $31-\mathrm{A} 1$ and Class 31-A2 pertrochanteric fractures were included in the analysis to allow for comparison with the existing literature. A single type of implant (i.e. PFNA nails) was utilised, which helped to prevent bias. Another strength of the study is its inclusion of postoperative ambulatory status at one year for all patients, a factor that was not reported in the aforementioned previous studies.

In conclusion, there were no significant differences in terms of complications, revision surgeries, union rates and ambulatory statuses between long and short cephalomedullary nails in the management of osteoporotic pertrochanteric fractures. A notable proportion of the patients were able to maintain ambulation status at one year following hip surgery, albeit not at their preinjury level. Both long and short nails provided safe and reliable outcomes for the treatment of osteoporotic pertrochanteric fractures.

\section{REFERENCES}

1. Johnell $O$, Kanis JA. An estimate of the worldwide prevalence and disability associated with osteoporotic fractures. Osteoporos Int 2006; 17:1726-33.

2. Cooper C, Campion G, Melton LJ 3rd. Hip fractures in the elderly: a worldwide projection. Osteoporos Int 1992; 2:285-9.

3. Johnell O, Kanis JA. An estimate of the worldwide prevalence, mortality and disability associated with hip fracture. Osteoporos Int 2004; 15:897-902.

4. Bjørgul K, Reikerås O. Outcome after treatment of complications of Gamma nailing: a prospective study of 554 trochanteric fractures. Acta Orthop 2007; 78:231-5.

5. Winnock de Grave P, Tampere T, Byn P, et al. Intramedullary fixation of intertrochanteric hip fractures: a comparison study of two implant designs. A prospective randomised clinical trial. Acta Orthop Belg 2012; 78:192-8.

6. Barton TM, Gleeson R, Topliss C, et al. A comparison of the long gamma nail with the sliding hip screw for the treatment of AO/OTA 31-A2 fractures of the proximal part of the femur: a prospective randomized trial. J Bone Joint Surg Am 2010; 92:792-8.

7. Kokoroghiannis C, Aktselis I, Deligeorgis A, et al. Evolving concepts of stability and intramedullary fixation of intertrochanteric fractures--a review. Injury 2012; 43:686-93.

8. Radcliff TA, Regan E, Cowper Ripley DC, Hutt E. Increased use of intramedullary nails for intertrochanteric proximal femoral fractures in veterans affairs hospitals: a comparative effectiveness study. J Bone Joint Surg Am 2012; 94:833-40.

9. Sehat K, Baker RP, Pattison G, et al. The use of the long gamma nail in proximal femoral fractures. Injury 2005; 36:1350-4.

10. Bellabarba C, Herscovici D Jr, Ricci WM. Percutaneous treatment of peritrochanteric fractures using the Gamma nail. Clin Orthop Relat Res 2000; 375:30-42.

11. Bojan AJ, Beimel C, Speitling A, et al. 3066 consecutive Gamma Nails. 12 years experience at a single centre. BMC Musculoskelet Disord 2010; $11: 133$.

12. Calvert PT. The Gamma nail--a significant advance or a passing fashion? J Bone Joint Surg Br 1992; 74:329-31.

13. Kukla C, Heinz T, Gaebler C, Heinze G, Vécsei V. The standard Gamma nail: a critical analysis of 1,000 cases. J Trauma 2001; 51:77-83.

14. Hou Z, Bowen TR, Irgit KS, et al. Treatment of pertrochanteric fractures (OTA 31-A1 and A2): long versus short cephalomedullary nailing. J Orthop Trauma 2013; 27:318-24.

15. Haidukewych GJ. Intertrochanteric fractures: ten tips to improve results. J Bone Joint Surg Am 2009; 91:712-9.

16. Kanis JA, Melton LJ 3rd, Christiansen C, Johnston CC, Khaltaev N. The diagnosis of osteoporosis. J Bone Miner Res 1994; 9:1137-41.

17. Kregor PJ, Obremskey WT, Kreder HJ, Swiontkowski MF; Evidence-based Orthopaedic Trauma Working Group. Unstable pertrochanteric femoral fractures. J Orthop Trauma 2005; 19:63-6.

18. Stern R. Are there advances in the treatment of extracapsular hip fractures in the elderly? Injury 2007; 38 Suppl 3:S77-87.

19. Boone C, Carlberg KN, Koueiter DM, et al. Short versus long intramedullary nails for treatment of intertrochanteric femur fractures (OTA 31-A1 and A2). J Orthop Trauma 2014; 28:e96-e100.

20. Kleweno C, Morgan J, Redshaw J, et al. Short versus long cephalomedullary nails for the treatment of intertrochanteric hip fractures in patients older than 65 years. J Orthop Trauma 2014; 28:391-7.

21. Marsh JL, Slongo TF, Agel J, et al. Fracture and dislocation classification compendium - 2007: Orthopaedic Trauma Association classification, database and outcomes committee. J Orthop Trauma 2007; 21(10 Suppl):S1-133.

22. Dripps RD. New classification of physical status. Anesthesiol 1963; $24: 111$.

23. Konstantinidis L, Papaioannou C, Blanke $P$, et al. Failure after osteosynthesis of trochanteric fractures. Where is the limit of osteoporosis? Osteoporos Int 2013; 24:2701-6.

24. Formiga F, Lopez-Soto A, Sacanella E, et al. Mortality and morbidity in nonagenarian patients following hip fracture surgery. Gerontology 2003; 49:41-5.

25. Kadowaki M, Kono M, Nishiguchi K, Kakimaru H, Uchio Y. Mortality in patients with hip fractures aged over 90 years: a report from a progressively aging island. Arch Gerontol Geriatr 2012; 54:e113-7.

26. Tay YW, Hong CC, Murphy D. Functional outcome and mortality in nonagenarians following hip fracture surgery. Arch Orthop Trauma Surg 2014; 134:765-72. 